\title{
A Cross Layer Approach for Efficient Multimedia Data Dissemination in VANETs
}

\author{
Boubakeur Moussaoui $^{\mathrm{a}, \mathrm{c}}$, Soufiene Djahel ${ }^{\mathrm{b}}$, Mohamed Smati $^{\mathrm{c}}$, John Murphy $^{\mathrm{d}}$ \\ a University of Constantine, Algeria \\ ${ }^{b}$ Manchester Metropolitan University, United Kingdom \\ ${ }^{c}$ ETA Laboratory, University of Bordj Bou Arreridj Algeria \\ ${ }^{d}$ UCD School of Computer Science and Informatics, Ireland
}

\begin{abstract}
The recent rapid expansion of multimedia applications spectrum, ranging from infotainment to healthcare and mission-critical scenarios, has incited researchers from different disciplines to develop various tools and protocols to support such applications. Many people use multimedia data while they travel in different transportation means and thus they need various QoS levels based on the type of application they are running. Such data is usually transmitted through 3G/LTE networks; however, the unprecedented increase of multimedia data volume makes satisfying their requirements in terms of low delay and higher bandwidth a challenge. Many experts foresee that Vehicular Ad-hoc Networks (VANETs) technology can be an efficient offloading solution for 3G/LTE networks by providing cost-free and robust data exchange between cars as well as among their passengers. Although VANETs pave the way toward several novel safety and non-safety applications that promise enhanced driving security and comfort, they suffer from several issues due to their intrinsic features such as higher vehicles mobility, rapidly changing topology, etc. In this work, we propose an Enhanced version of AODV (En-AODV) protocol to deal with routes instability issue in VANETs. En-AODV leverages cross-layer information on the link quality combined with the knowledge of the final destination of the receiver vehicle to establish more stable routes. The obtained simulation results confirm the efficiency of En-AODV and highlight its supremacy over AODV under various metrics and scenarios. En-AODV has particularly proven its ability to establish stable routes while significantly reduce the overhead generated by control packets, freeing up the channel to carry more data packets.
\end{abstract}

Keywords: Routing protocols, AODV, VANETs, Multimedia applications, Cross layer.

\section{Introduction}

In June 2016, Cisco predicts that IP video will represent 82 percent of all traffic by 2020, up from 70 percent in 2015 [1]. This expected increase of multimedia traffic is a result of the increasing popularity of multimedia applications among users of different age classes for different purposes (e.g. entertainment, VoIP and IP telephony, remote surgery, video conferencing etc.). A typical multimedia application can be classified as either streaming stored audio/video, conversational voice/videoover-IP, or streaming live audio/video [2]. When designing networked multimedia applications, we must meticulously take into account their specific constraints and requirements in order to achieve the sought QoS (Quality of Service) level. The most important requirements of these applications are the high bit-rate, low delay and packet loss rate. To meet these stringent requirements in a connected vehicles system, Vehicular Ad-hoc Networks (VANETs) routing protocols must be designed in a way that ensures longer stability of the established routes and larger bandwidth, so that the end-to-end delay is reduced and packets loss is minimized. Achieving this goal makes VANETs

Email addresses: b.moussaoui@univ-bba.dz (Boubakeur Moussaoui), s.djahel@mmu.ac.uk (Soufiene Djahel),

m. smati@univ-bba.dz (Mohamed Smati), j.murphy@ucd.ie (John Murphy) an efficient offloading solution for 3G/LTE networks which are currently the main carrier of multimedia data.

VANETs technology promises to provide various services to both drivers and passengers and help road traffic authorities for better control and mitigation of traffic congestion, thus reducing air pollution and the number of accidents on the roads [3]. In this work, we focus on infotainment services that provide the passengers with the capability to communicate with other passengers traveling in other vehicles, sharing multimedia content, playing interactive games etc. Unfortunately, the inherent characteristics of VANETs, such as high speed of vehicles, frequent link failure and bandwidth scarcity, make it difficult to accommodate the needs of the aforementioned infotainment services. Therefore, novel routing protocols are needed to provide more stable routes to support the exchange of infotainment applications data.

Despite the numerous protocols proposed in the literature to find the most stable routes for carrying packets between two communicating nodes, their performance is still far from the sought level of QoS. We can distinguish two major classes of such routing protocols: topology based and position based protocols. The former class can be further split into two other subclasses; proactive and reactive protocols. Ad-hoc On-demand Distance Vector (AODV) is a reactive routing protocol designed to establish communication routes in Mobile Ad-hoc Networks 
(MANETs). This protocol finds the shortest path to route data packets among communicating nodes. In this paper, we investigate routes instability issue in VANETs, which is mainly due to their aforementioned intrinsic characteristics, and aim to overcome it. To this end, we propose an original enhancement to AODV protocol dubbed En-AODV (Enhanced AODV) in order to achieve the following: (i) establish the most stable path relaying two communicating vehicles (ii) and quickly replacing any broken link in this path by an alternative link(s) of a good quality. En-AODV exploits cross-layer information about the link quality (i.e. its estimated lifetime) jointly with the introduced concept of Destination Region to establish more stable routes. Compared to AODV, our protocol significantly reduces the RREQs overhead, thanks to the destination region field added to RREQs packets, and establishes more stable routes, thanks to the more accurate lifetime estimation function, with faster replacement of lost links.

The rest of this paper is organized as follows. Section II reviews the literature. In Section III, we provide a detailed description of our proposed enhancement to AODV. Section IV presents the simulation setting and discusses the obtained results. Finally, we conclude the paper in Section V.

\section{Related work}

Several protocols have been proposed in the literature to address the routing problem in VANETs. Each of them has somewhat enhanced the routing function, with respect to one or more metrics, and has its own advantages and drawbacks. Ad-hoc On-demand Distance Vector (AODV) protocol is one of the most popular routing protocols that has been subject to several attempts of improvement in recent years, as described below. These efforts focused mainly on how to adapt it to VANETs environment since it was originally designed for MANETs (Mobile Ad-hoc Networks).

In order to adapt AODV to VANETs [4] proposed a crosslayer approach in which the End-to-End (E2E) delay of each path is used as a key metric for the best route selection. Moreover, a realistic radio propagation model, called Communication Ray tracer (CRT) developed at the SIC-XLIM laboratory, was added to ns 2 packages to get more accurate evaluation results. Although using cross layer metrics can yield more accurate estimation of the E2E delay, the authors measured the transmission delay over each link rather than its remaining lifetime, which is more important in VANETs to ensure faster and efficient reaction to sudden links failure.

In [5], the route reliability was defined based on vehicles movement information (position, velocity, direction ...) along with the channel state information and transmit power. The authors calculate also the link expiration time, which denotes the time during which the link is still valid. The obtained simulation results have proven the efficiency of this proposal in minimizing the packet loss rate and decreasing the E2E delay. Note that vehicles unique motion and speed are important metrics to consider in the routing function as they directly affect the channel state, leading to loss of connectivity. However, other metrics, such as the congestion level, can have devastating impact on the channel state and lead to frequent links failure; therefore appropriate congestion avoidance measures are also needed.

Similarly to [7], the main idea of [6] consists in using adjacent vehicles as probabilistic relays of unsuccessful data packets transmission, however in this latter the relay node is not compulsory to be a base-station. When a vehicle perceives that an adjacent source vehicle has sent a packet but no acknowledgment was received from the destination, it relays the packet to avoid multiple unsuccessful retransmissions. This led to an improvement of AODV and Ad-hoc On-demand Multipath Distance Vector (AOMDV) performance.

In [8], a new scheme was proposed to improve AODV routing efficiency for multimedia applications in VANETs. In this scheme, the road network is divided into non-overlapping sections (segments) where the connectivity of each vehicle within its segment is provided by a neighbor table stored in its memory. Vehicles located at intersections are called gateways. They use road segments weight values, defined based on the Quality of Services (QoS) required by an application, to select the best available route. In this protocol, the vehicles broadcast asynchronously link requests to discover their neighbors and then share their neighbor tables with other vehicles within the same road segment. In this way, the additional generated traffic will consume the limited available bandwidth. Furthermore, predicting the link availability time using inter-vehicles distance and their velocity only is not the most accurate way.

[9] proposes to combine AODV basic mechanism with a geographic routing approach. In this work, the source vehicle modifies the RREQ packet format by adding the recent known position of the destination vehicle and a time-to live (TTL) value to limit the number of hops this packet will traverse. In this way, the RREQ will reach its intended destination without any unnecessary flooding of the network. Another work presented in [10] proposes to leverage the vehicle speed, its acceleration, direction and link quality between vehicles to compute a total weight for each route (TWR). The TWR value along with an expiration time estimation for each route will determine the best route. The route discovery phase is similar to AODV, with a slight modification in RREQ and RREP packets format.

In [11], the authors designed a scheme that takes into account the interference level to establish routes suitable for relaying live video streaming packets over VANETs. This scheme aims to maximize the end-to-end video delivery quality by seeking the best trade-off between distortion and delay. Other works, such as [12] and [13], have focused on estimating the available bandwidth for each candidate route, then choosing the route that maximizes this resource to support the applications requiring large bandwidth to run properly.

The authors of [14] investigated the major challenges hindering the achievement of efficient routing in VANETs by analysing data collected from real-work experiments. Then, they proposed a novel multihop routing protocol wherein three factors are used for route selection: transmission rate, vehicle mobility, and number of hops. The novelty of this protocol consists in employing a Q-learning algorithm to estimate the transmission rate from the received hello packets, and a fuzzy logic based algorithm for route selection. The performance of the 
proposed protocol was evaluated in real VANET environment as well as via simulation (to assess its scalability) and the obtained results were very promising.

Achieving high packet delivery ratio with reduced overhead and delay were the major goals of the work presented in [15]. In this work, an original protocol combining the concept of backbone vehicle with network coding was proposed. First, backbone vehicles are chosen based on vehicles movement dynamics and links quality. Afterwards, interflow network coding is applied at the backbone vehicles, significantly reducing the number of generated packets by up to $25 \%$ compared with the existing protocols.

\section{Proposed solution}

In this section, we present the main idea of our proposed protocol named En-AODV (Enhanced AODV) aiming at finding the most suitable inter-vehicle routes that satisfy multimedia applications requirements. To this end, En-AODV has two major goals to achieve: (i) finding the most stable path relaying the source and destination vehicles and (ii) quickly react to the occurrence of a link failure in this path and provide an alternative $\operatorname{link}(\mathrm{s})$ of good quality. Moreover, it is worth to mention that although En-AODV mainly operates at the network level where the routing function is performed, it has a cross layer aspect which consists in the cooperation between the physical and network layers to select the most stable path among the available options. This is achieved by exploiting a physical layer measurement (i.e. the received signal strength value) at the routing level to estimate a given link lifetime.

\subsection{Motivation}

Designing an efficient routing protocol in MANETs means providing the best available path between two communicating nodes based on a set of metrics. In such a protocol, the route search phase should take into account all the constraints imposed by the network (e.g. nodes speed, available bandwidth, transmission range, etc.) as well as the target application requirements, such as rapid response and transmission reliability. These constraints are usually met by the most stable path that lasts for the maximum possible duration.

The high speed of vehicles and rapidly changing topology in VANETs makes the above task of routing more challenging, as faster and more efficient reaction (i.e. maintenance task to replace the broken link) from routing protocols, in case of links failure, is mandatory to support the numerous time-sensitive and bandwidth-hungry applications running over VANETs. Indeed, the maintenance scheme is a crucial component of any routing protocol in VANETs as, if it is not designed properly, it can lead to the following issues: (i) the creation of bottlenecks in the network as a consequence of the large number of control packets used in route discovery phase in reactive protocols (e.g., AODV); (ii) increase of the E2E delay, and (iii) increase in packets loss, especially during the maintaining phase.

To overcome the aforementioned issues, we propose in this paper En-AODV whose detailed operations are presented in the following subsections.

\subsection{Reducing the number of control packets}

In order to reduce the number of broadcasted control packets in VANETs, we exploit the information on vehicles movement (i.e., recent known position and final destination) in addition to the digital map of the road network. We assume that the city digital map is divided into multiple regions according to the postal zones, and all the vehicles are aware of this configuration of the city map. By discovering the location of the final destination, we aim to minimize the number of vehicles that will rebroadcast the received RREQs, thereby significantly reducing the communication overhead and ensuring better resources utilization. For example, as shown in Figure 1, we suppose that the destination vehicle moves from region 3 to region 4; if an intermediate vehicle receives its corresponding RREQ, it extracts the destination region value from the packet header, shown in Figure 3, and broadcasts the RREQ only if its current location is Region 4 or it is moving towards it, otherwise it discards it.

It is worth mentioning that the source vehicle can discover the region (or zone) where the final destination of the target vehicle is located through the Traffic Management System (TMS) [16]. It is acknowledged that modern TMSs can provide optimal (shortest or fastest) routes for all vehicles in the road network. In smarter cities context, we foresee that each vehicle requests an optimal route to its intended destination from the TMS before starting its journey. Consequently, the TMS becomes aware of the destination region of each single vehicle in VANETs and can make such information available to any other vehicle upon request. To prevent any security and privacy issue, the TMS will not provide an accurate location of a vehicle destination but instead a wider region, which is sufficient for our protocol to function efficiently.

\subsection{Link lifetime estimation}

A route connecting two distant vehicles consists of multiple links (i.e. several vehicles). Usually, the main metric used to choose such a route is the number of hops, which unfortunately might be interrupted rapidly due to VANETs features discussed earlier. In En-AODV, we use additional metrics to guarantee the selection of the most stable route. This is achieved by designing a novel link lifetime estimation scheme. This latter predicts the lifetime of each link composing a candidate route, and the lifetime of the whole route is defined as the shortest lifetime among its whole links.

In our scheme, we make use of lower layers information (i.e. the physical layer) to accurately calculate the expected duration for which the communication between two neighbor vehicles still exist. Furthermore, to make our scheme more realistic, we use the information available in a smart vehicle and the power of the received signal, which can be calculated at the reception of a packet.

The formula which defines the relation between the power of a received signal and the current distance separating two communicating vehicles is:

$$
\operatorname{Pr}=\operatorname{Pt.Gt.Gr} \cdot\left(\frac{\lambda}{4 \pi R}\right)^{2}
$$




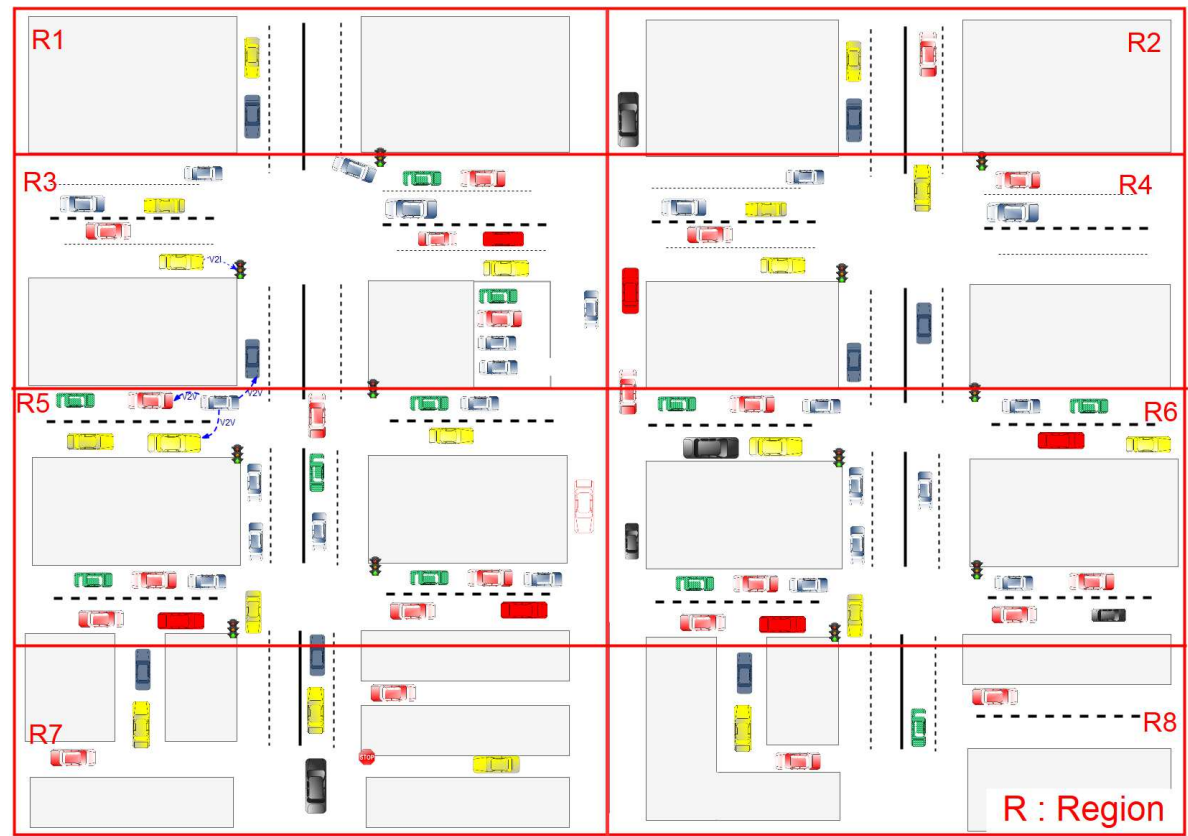

Figure 1: Example of an urban road network organized in 8 regions

Where :

$\mathrm{Pt}$ : refers to the power of the signal at the emission measured in Watt.

Gt : is the transmitter gain (antenna gain) measured in decibel.

Gr : denotes the receiver gain (antenna gain) measured in decibel.

$\mathrm{R}:$ is the distance $(\mathrm{m})$.

$\mathrm{L}$ : represents the system loss.

$\lambda:$ is the wavelength $(\mathrm{m})$.

From Eq. (1), the distance between two communicating vehicles can be calculated as follows:

$$
R=\left(\frac{\lambda}{4 \pi}\right) \sqrt{\frac{P t \cdot G t \cdot G r}{P r}}
$$

To ensure that the communication can be established between two communicating vehicles, the power of the received signal must be greater than a threshold value Ps, (which is usually very small because receivers are very sensitive, we set Ps = $\mathrm{Pt} / 2$ and use it as a threshold in our simulation). Therefore, we have:

$$
P r \geq P s
$$

By replacing the value of Pr from (1) in (3), we get:

$$
\text { Pt.Gt.Gr. }\left(\frac{\lambda}{4 \pi R}\right)^{2} \geq P s
$$

By simplifying this formula, we deduce the maximum distance within which two vehicles can communicate correctly:

$$
R \leq\left(\frac{\lambda}{4 \pi}\right) \sqrt{\frac{P t \cdot G t \cdot G r}{P s}}
$$

In other words, the relative distance between the sender and receiver is given as follows:

$$
R=V r \cdot T
$$

Where Vr represents the relative velocity between vehicles, and $\mathrm{T}$ represents the time.

$\mathrm{Vr}$ can be written as:

$$
V r=\|\overrightarrow{V s}-\overrightarrow{V R}\|
$$

VS and VR are the sender and receiver velocities, respectively.

By using Eq.(4) and Eq.(5), we infer that:

$$
V r . t \leq\left(\frac{\lambda}{4 \pi}\right) \sqrt{\frac{P t . G t \cdot G r}{P s}}
$$

After simplification, we can deduce the maximum time of communication (i.e. the link lifetime) as:

$$
t \leq\left(\frac{\lambda}{4 \pi \cdot V r}\right) \sqrt{\frac{P t \cdot G t \cdot G r}{P s}}
$$

Here, we consider using an isotropic antenna that emits its power with the same manner in all space directions, thereby the gain will be equal to 1 or ignored.

It is worth to mention that the relative velocity depends on the velocity vectors of both sender and receiver vehicles. In 

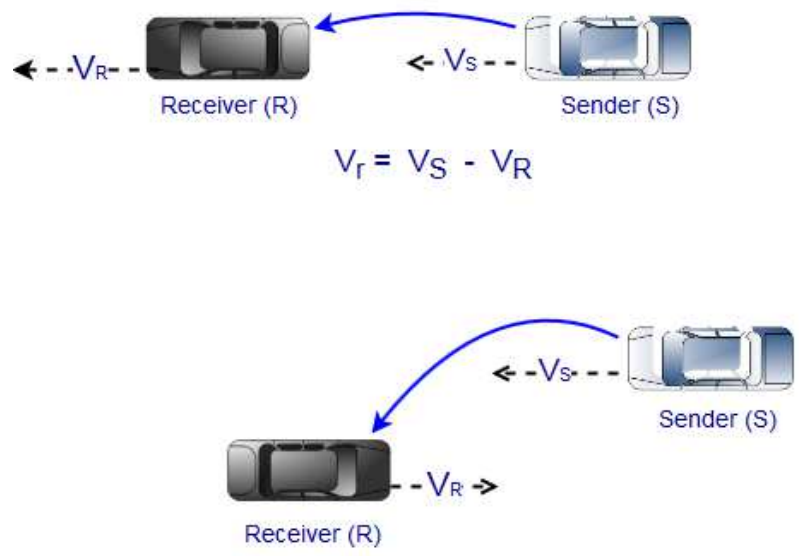

$$
V_{r}=V_{S}+V_{R}
$$

Figure 2: Illustration of the relative velocity between the sender and receiver vehicles

Figure 2, we illustrate two different scenarios where the sender and the receiver move in the same or opposite direction within the same road lane and their velocity vectors are parallel. Other cases where the sender and receiver velocity vectors are not parallel and separated by an angle (i.e. the sender and receiver are approaching an intersection from different directions) are not shown here but we have taken into consideration this case in our simulation.

\subsection{Defining new format of control packets}

The new RREQ packet, used in our En-AODV, contains two additional fields besides those used by AODV, as shown in Figure 3. The first field represents the minimum lifetime (MLF) of a route, which can be updated only if an intermediate vehicle finds that the lifetime of the link relaying it to the preceding vehicle in that route is lower than the value in the packet. This update action is necessary to ensure that the destination vehicle gets an accurate shortest lifetime value for the route taken by the received RREQ; thereby more accurate selection of the RREP route can be made.The second field, named Destination Region (DR) contains information about the region toward which the destination vehicle is heading. Upon reception of a RREQ packet by an intermediate vehicle, it rebroadcasts it only if the value of the Destination Region field is similar to the vehicle current or destination region, otherwise this packet is discarded.

Once the RREQ packet reaches its final destination, a RREP packet is sent back to the originator vehicle. As shown in Figure 4, an additional field is piggybacked to RREP to inform the source and intermediate vehicles about the lifetime offered by this route. The destination vehicle chooses the route with the longest lifetime among the available routes, then it unicasts a route reply to the source vehicle through the chosen route.

\subsection{Route search phase}

When a vehicle needs to send multimedia packets toward another distant vehicle, it checks first if a valid route to this vehicle exists in its routing table, if it is the case it then starts transmitting the packets using this route, otherwise it imitates a new route search phase. This latter phase involves broadcasting a new RREQ to all its neighbors in the same manner as in AODV, except that here two additional fields are added. Note that the initial value of the minimum lifetime field is set to zero so that the first receiver vehicles (i.e. the 1-hop neighbors of the sender) will update it with the lifetime of the link relaying them to the originator of the RREQ. Every intermediate receiver of this RREQ rebroadcasts it only if its current/destination region matches the value of the Destination Region field; otherwise, it refrains from doing so. In the former case, the vehicle should update the minimum lifetime (see Section 3.3) field if necessary before rebroadcasting the RREQ packet.

Each intermediate vehicle involved in the search procedure, before rebroadcasting the RREQ if the above condition is met, should check the existence of a valid fresh route to the destination in its routing table; if such a route exists, it then changes the value of the Type field to unicast and sends the RREQ packet to the destination via the existing route, instead of rebroadcasting it.If another RREQ with the same identifier and source address is received by the same intermediate vehicle, it first checks if the new minimum lifetime value is greater than the existing value in the corresponding routing table entry. If it is the case then it rebroadcasts the new RREQ and update its routing table entry (Next hop to reach the originating vehicle (i.e. the reverse route) and minimum lifetime value). The destination vehicle waits an arbitrary time to receive some RREQs and update their lifetime values, if necessary, using the same mechanism discussed in section 3.3. Afterwards, it compares the RREQs, if there is more than one, and choose the route with the longest lifetime to unicast the RREP back to the source vehicle. Note here, as opposed to AODV, only the destination vehicle is allowed to send the RREP packets. The reason behind this is to prevent missing more stable routes that might not be known by some intermediate vehicles at the time of receiving the RREQ.

As the RREQ propagates via the network, each receiver intermediate vehicle adds a new entry to its routing table for the source vehicle and sets its lifetime to the minimum value between the received value in the RREQ and that measured with its neighbor (i.e. the sender of the RREQ). Subsequently, when the corresponding RREP reaches this vehicle it adds a new entry for the originator vehicle (i.e. the destination of the RREQ) and sets its lifetime to the value indicated in the minimum lifetime field.

\subsection{Route maintenance phase}

The second challenge in En-AODV is the maintenance of the established route since the high speed of vehicles leads to a rapid change of the network topology. Although we estimate, in this work, the lifetime of the entire route, the unexpected behavior (e.g. sudden change of direction or velocity) of drivers in VANETs can result in a link failure before reaching its estimated lifetime. Therefore, in this case a fast and efficient reaction is needed to replace the broken link(s). Such a broken link is detected using periodic hello messages exchanged between neighboring vehicles. A route maintenance process is initiated only if the remaining lifetime of the route is lower than 


\begin{tabular}{|c|c|c|c|c|c|c|c|c|c|}
8 & 5 & 8 & 32 & 32 & 32 & 32 & 32 & 32 \\
\hline Type & Flags & Hop Count & RREQ ID & $\begin{array}{c}\text { Destination } \\
\text { Addresse }\end{array}$ & $\begin{array}{c}\text { Destination } \\
\text { Seq Nbr }\end{array}$ & $\begin{array}{c}\text { Source } \\
\text { Addresse }\end{array}$ & $\begin{array}{c}\text { Source } \\
\text { Seq Nbr }\end{array}$ & $\begin{array}{c}\text { Minimum } \\
\text { Lifetime }\end{array}$ & $\begin{array}{c}\text { Destination } \\
\text { Region }\end{array}$ \\
\hline
\end{tabular}

Figure 3: Format of the new RREQ packet

\begin{tabular}{|l|c|c|c|c|c|c|c|c|}
8 & \multicolumn{2}{c}{5} & 32 & 32 & 32 & 32 & 32 & 32 \\
\hline Type & Flags & Hop Count & $\begin{array}{c}\text { Prefix } \\
\text { Size }\end{array}$ & $\begin{array}{c}\text { Destination } \\
\text { Addresse }\end{array}$ & $\begin{array}{c}\text { Destination } \\
\text { Seq Nbr }\end{array}$ & $\begin{array}{c}\text { Source } \\
\text { Addresse }\end{array}$ & Lifetime & $\begin{array}{l}\text { Minimum } \\
\text { Lifetime }\end{array}$ \\
\hline
\end{tabular}

Figure 4: Format of the new RREP packet

a given threshold, otherwise the source vehicle will establish a new route to communicate with the destination if necessary.

To replace a broken link (e.g. the link between C and D shown in Figure 5) belonging to an established route between two vehicles, either $C$ or D sends a RREQ to establish a new route with the source (for the node $\mathrm{D}$ ) or with the destination (for the node $\mathrm{C}$ ), if the following inequality is verified:

$$
\text { Nhops }<\left(L \_ \text {path } / 2\right)+1
$$

Where:

Nhops: is the number of hops between the vehicle C (D) and the destination (source) vehicle.

L_Path: refers to the length of the route in term of number of hops.

Notice that each vehicle can learn the Nhops and L_Path values from the Hop Count field of the received RREQ and its corresponding RREP packet. In the special case where the Nhops value calculated by both ends (e.g. C and D) of the broken link are equal, then the node with the lower identifier generates the RREQ.

A summary of the different operations of the route search and maintenance phases is presented in Algorithms 1 and 2, respectively.

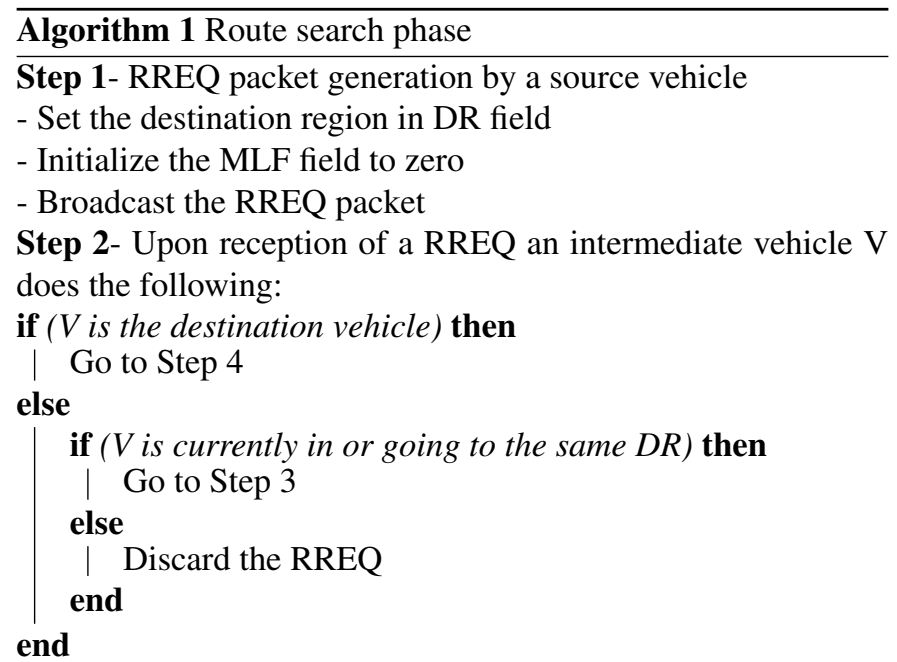

Step 3- Compute the remaining link lifetime (LF) with the sender vehicle (neighbor) using Eq. (7)

if $(M L F>$ computed $L F)$ then

Update the MLF value in the RREQ with the computed LF Broadcast the updated RREQ

else

Broadcast the received RREQ

end

Step 4- Wait an arbitrary time to receive more RREQs

- Select the route with max MLF value

- Send a RREP to the source node using the chosen route 


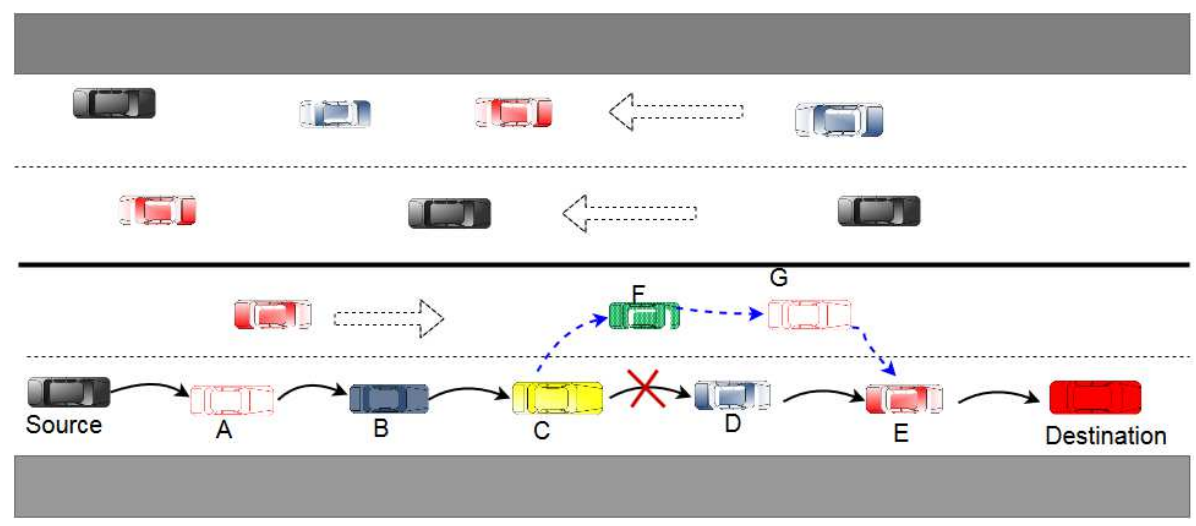

Figure 5: Example of a broken link in a route connecting two vehicles

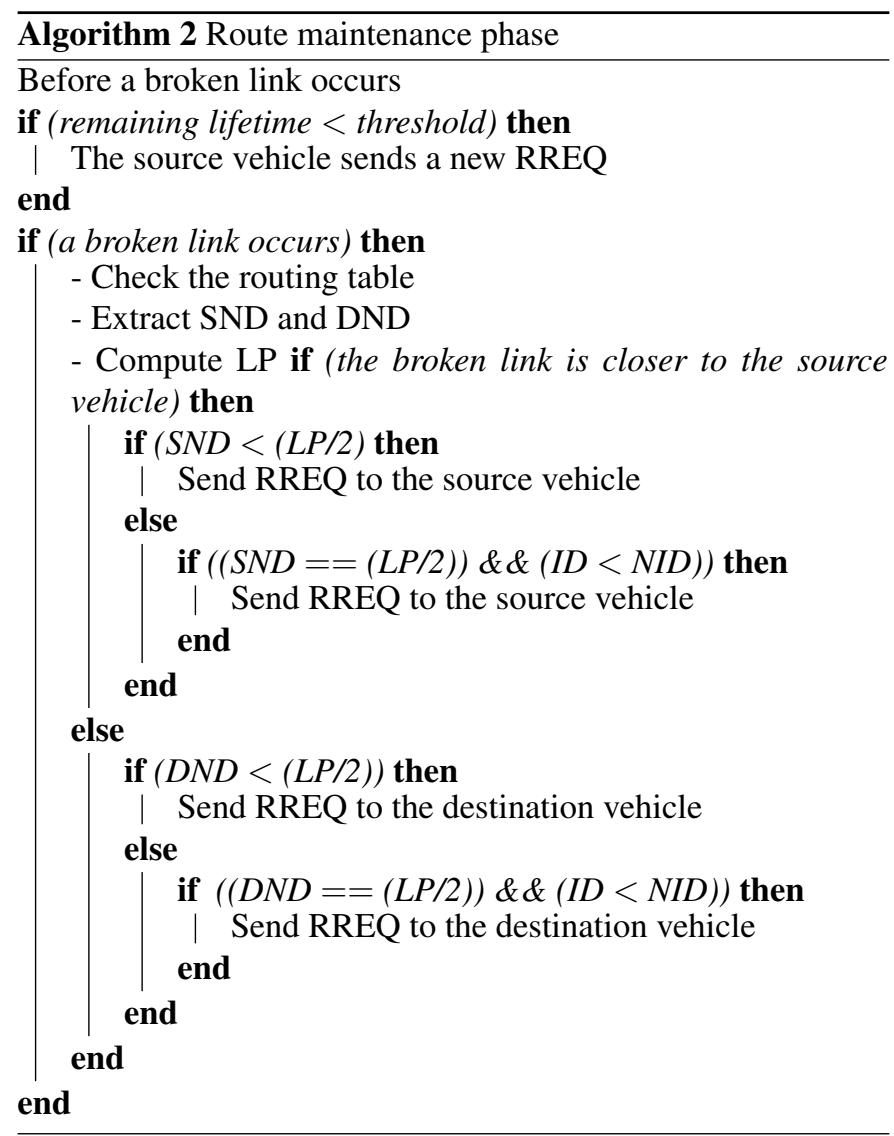

Where:

ID: is the identifier of current vehicle.

NID: is the identifier of the neighbor vehicle.

LP: represents the length of the route.

SND: refers to the distance between the current vehicle and the source vehicle.

DND: refers to the distance separating the current vehicle from the destination vehicle.

\section{Performance evaluation}

Our simulation was conducted using ns-3 network simulator and the microscopic road traffic simulator SUMO. Ns-3 is chosen to simulate the different protocols (AODV, En-AODV with varying number of regions), whereas SUMO is used to generate realistic mobility traces of vehicles to mimic a real world road network. We have generated a realistic mobility model using the tool "Mobility Model Generator for Vehicular Networks" (MOVE, version 2.7.1) introduced in [17]; SUMO is then used to simulate realistic road traffic scenarios. The Digital Map used is $2000 \mathrm{~m}$ × $2000 \mathrm{~m}$ wherein the distance between two successive intersections is $300 \mathrm{~m}$ and each road segment has two lanes. The maximum velocity of a vehicle is set to 16.66 $\mathrm{m} / \mathrm{s}$ (i.e. $60 \mathrm{Km} / \mathrm{h}$ ). IEEE802.11p MAC layer [18] is also used with channel bandwidth of $10 \mathrm{MHz}$. During our simulation, 10 scenarios were tested for each protocol using the same digital maps. The different values of essential simulation parameters are given in Table 1.

To evaluate the effectiveness of our proposed En-AODV we will compare its performance, via simulation, against the baseline AODV protocol. The metrics used for this comparison are; Packet Delivery Ratio (PDR), End-to-End (E2E) delay, and the number of RREQs broadcasted in the network to assess the overhead generated by these packets. This latter metric is very important as reducing it will save more bandwidth that can be used to carry effective data.

The above metrics are defined as follows.

$$
\begin{aligned}
P D R & =\frac{\text { No. of success fully received packets }}{\text { Total No. of transmitted packets }} \\
E 2 E \text { delay } & =\text { reception time }- \text { emission time }(\mathrm{ms})
\end{aligned}
$$

For this metric, we compute the average value of all the packets in the same scenario.

The total number of broadcasted (and re-broadcasted) RREQ packets by each node is defined as follows:

$$
\# R R E Q=\sum_{\text {No.of vehicles }} \text { Total No. of sent RREQs }
$$

In Figure 6, we show the achieved PDR under varying number of vehicles in VANETs. The plotted results indicate that our 
protocol outperforms AODV by almost $15 \%$ in average. We observe also that the number of regions defined in the road network has an influence on the achieved PDR when the network density changes. In low dense networks (\#vehicles $<75$ ), the lower the number of regions is the higher PDR we get. In contrast, when the network density increases (\#vehicles $>100$ ), a higher number of regions yields better results. This is because establishing a stable route in sparse network is very challenging, thus a reduced number of regions makes the vehicles rebroadcasting more RREQs to establish routes. However, in dense networks, a higher number of regions is more suitable as in this case establishing routes is less challenging and more regions will reduce the RREQs overhead.

To further explain the above results, Figure 7 illustrates the number of broadcasted RREQs in VANETs under varying network size. Here, the plotted total number of RREQs gives more insights on what is happening during communication. A higher number of the broadcasted RREQs means links failure is more frequent and more bandwidth is consumed. Moreover, the results suggest that this number is proportional with the increase of VANET's size and that En-AODV reduce this number by up to $60 \%$ (when the number of vehicles is 120 ).

\begin{tabular}{|c|c|}
\hline Parameter & Value \\
\hline Frequency band & $5.150 \mathrm{e} 9 \mathrm{~Hz}$ \\
\hline Bandwidth & $10 \mathrm{MHz}$ \\
\hline Data rate & $6 \mathrm{Mbits} / \mathrm{s}$ \\
\hline Transmission range & $\sim 350 \mathrm{~m}$ \\
\hline No. of vehicles & $20 . .150 \mathrm{vehicles}$ \\
\hline Vehicles max velocity & $16.66 \mathrm{~m} / \mathrm{s}$ \\
\hline Traffic flows & $5 . .25 \mathrm{CBR}$ flows \\
\hline Sending rate of data packets & 30 packets/s \\
(per flow) & \\
\hline Packet size & $1024 \mathrm{bytes}$ \\
\hline Network size & $2000 * 2000 \mathrm{~m}^{2}$ \\
\hline No. of regions & 4,8 \\
\hline PI & 3.1415926535897 \\
\hline Transmission antenna gain & 1 \\
\hline Received antenna gain & 1 \\
\hline Speed of light in vacuum & $299792458.0 \mathrm{~m} / \mathrm{s}$ \\
\hline Transmission power & $16.0206 \mathrm{dbm}$ \\
\hline Tx power threshold (min) & $-96.0 \mathrm{dbm}$ \\
\hline
\end{tabular}

Table 1: Simulation parameters setting

Since multimedia applications are very sensitive to data packets E2E delay, we measure in Figure 8 the impact of VANET's size on this metric under the baseline AODV as well as the two versions of En-AODV (i.e. 4 and 8 regions). As we can see, En-AODV ensures a significantly lower average E2E delay compared to AODV, and this performance is further improved under higher number of regions. Notice that when VANET's size is lower than 40 vehicles the average E2E delay is high due to the limited number of available routes and the longer distance between the vehicles, which leads to unreliable transmissions and thus increase the packets transmission de- lay. This situation improves gradually when VANET becomes denser, as more routes are available with different lengths and lifetimes, and hence the better achieved E2E delay.

\section{Conclusion}

The recent advancements in communication technologies have paved the way towards the emergence of smart vehicles capable of communicating with each other through VANETs. This, therefore, led to the expansion of the number of applications running over VANETs such as multimedia applications used for infotainment and other purposes. To support this category of applications, we have proposed En-AODV protocol that provides stable routes required for any communicating vehicles in VANETs. The key idea behind this protocol is to exploit cross layer information about the quality of the link and its expected lifetime in addition to the reduction of the communication overhead, thanks to the destination region information. Simulation results using ns-3 show that En-AODV exhibits better performance than AODV. As a future work, we intend to use Road Side Units (RSUs) deployed along the road network to handle critical situations such as (i) sparse network or empty road segments or (ii) uncooperative behaviors of some drivers.

\section{References}

[1] White paper: Cisco VNI Forecast and Methodology, 2015-2020, [Online]. http://www.cisco.com/c/en/us/solutions/collateral/serviceprovider/visual-networking-index-vni/complete-white-paper-c11481360.html.

[2] J-F. Kurose and K-W. Ross, Computer Networking, A Top-Down Approach. Sixth Edition. Pearson Education 2013. ISBN-13: 978-0-13285620-1, ISBN-10: 0-13-285620-4.

[3] S. Djahel, R. Doolan, G-M Muntean and J. Murphy. A Communicationsoriented Perspective on Traffic Management Systems for Smart Cities: Challenges and Innovative Approaches. IEEE Communications Surveys and Tutorials, Vol. 17, No. 1, 2015.

[4] J. LEDY, H. BOEGLEN, B. HILT, H. Abouaissa and R. Vauzelle. An enhanced AODV protocol for VANETs with realistic radio propagation model validation. In Intelligent Transport Systems Telecommunications, (ITST), 9th International Conference on. IEEE, 2009. p. 398-402.

[5] Y. HE, W. XU and X. LIN. A Stable Routing Protocol for Highway Mobility over Vehicular Ad-Hoc Networks. In IEEE Vehicular Technology Conference (VTC Spring), 2015. p. 1-5.

[6] R. Anggoro, T. Kitasuka, R. Nakamura and M. Aritsugi. Performance evaluation of AODV and AOMDV with probabilistic relay in VANET environments. In the Third IEEE International Conference on Networking and Computing (ICNC), 2012. p. 259-263.

[7] A. Balasubramanian, R. Mahajan, A. Venkataramani, B.N. Levine, and J. Zahorjan. Interactive WiFi Connectivity for Moving Vehicles. Proc. of ACM SIGCOMM 08, pp. 427-438, Seattle, Washington, USA, August 2008.

[8] Z. AMJAD, and W-C. SONG. Road aware QoS routing in VANETs. In Network Operations and Management Symposium (APNOMS), 2015. p. 133-138.

[9] M. Al-Rabayah and R. Malaney, A New Scalable Hybrid Routing Protocol for VANETs. IEEE Transactions On Vehicular Technology, Vol. 61, No. 6, July 2012.

[10] X. Yu, H. GUO and W-C. Wong. A reliable routing protocol for VANET communications. In the seventh IEEE International Wireless Communications and Mobile Computing Conference, 2011. p. 1748-1753.

[11] H. Wu and H. Ma. Opportunistic routing for Live Video Streaming in vehicular ad hoc networks. In IEEE 15th International Symposium on World of Wireless, Mobile and Multimedia Networks (WoWMoM), 2014. p. 1-3. 


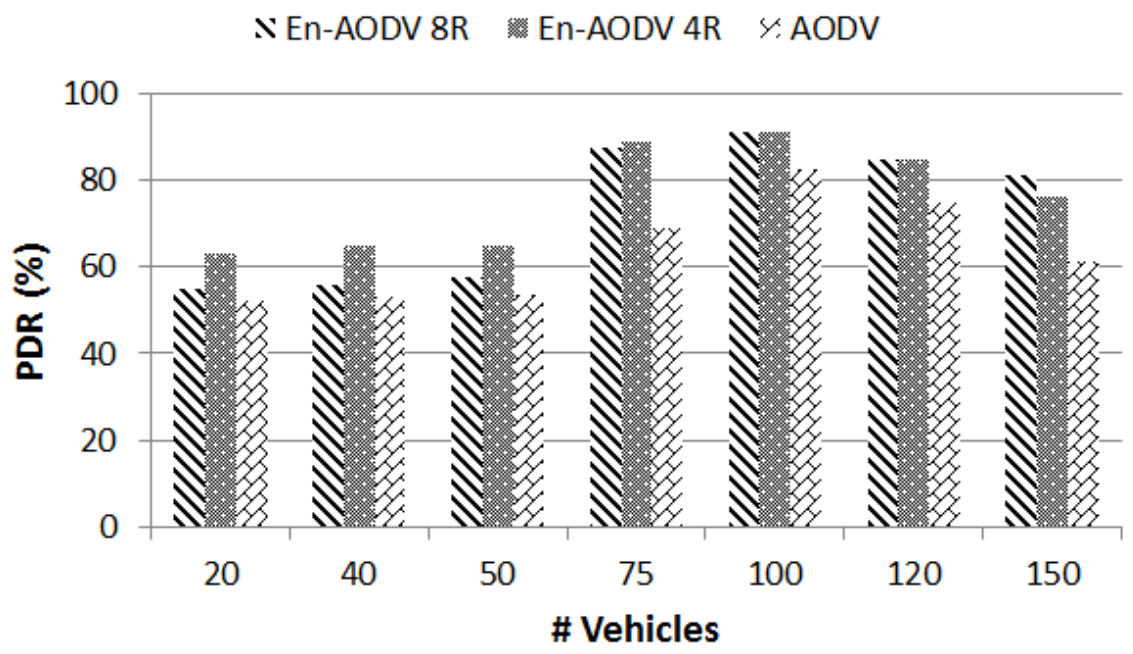

Figure 6: Impact of VANET's size on the PDR

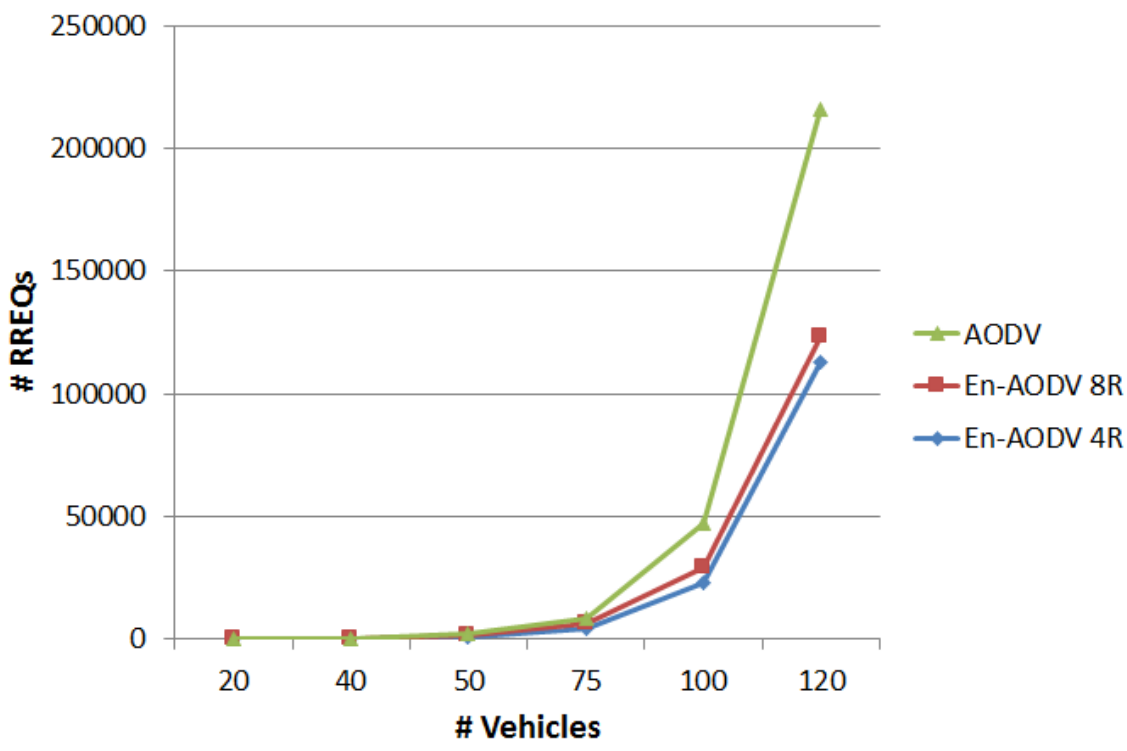

Figure 7: Variation of the number of broadcasted RREQs under varying VANET's size 


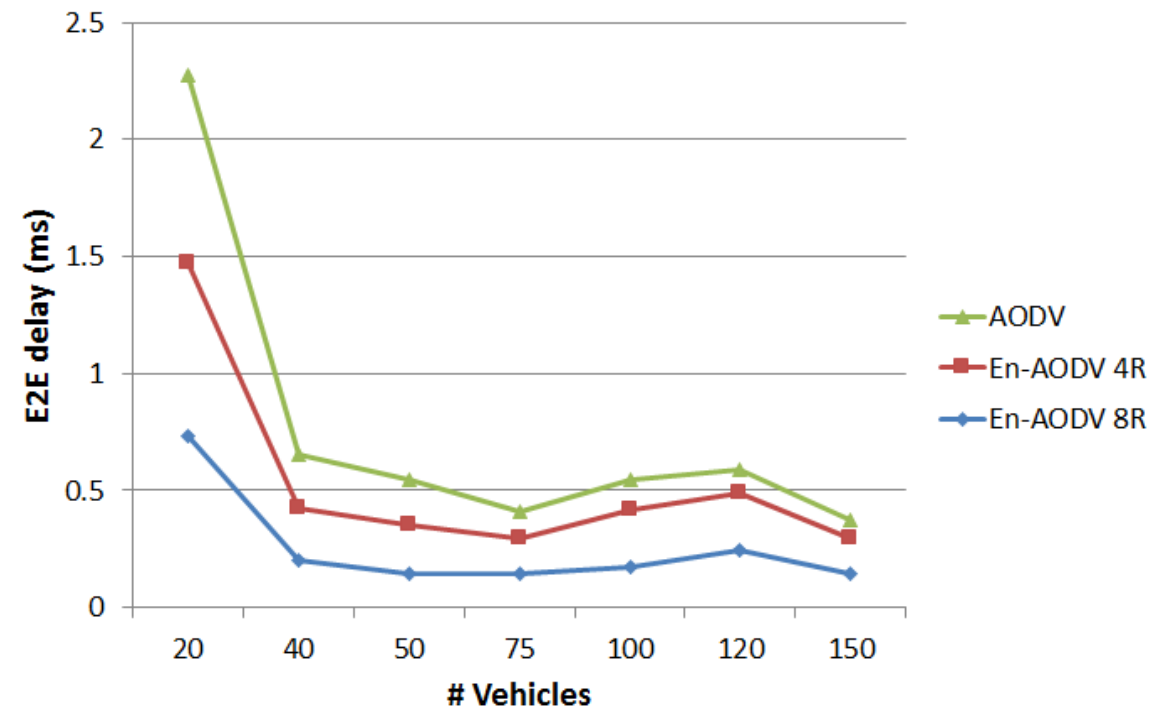

Figure 8: Averages E2D delay vs. VANET's size

[12] C-T. Barba, A-M. Mezher, M-A. Igartua, I. Guerin-Lassous and C. Sarr. Available bandwidth-aware routing in Urban Vehicular ad-hoc networks. In IEEE Vehicular Technology Conference (VTC Fall), 2012. p. 1-5.

[13] C. SARR, C. CHAUDET, G. CHELIUS, I. Guerin-Lassous. Bandwidth estimation for IEEE 802.11-based ad hoc networks. IEEE transactions on Mobile Computing, 2008, vol. 7, no 10, p. 1228-1241.

[14] C. Wu, Y. Ji, F. Liu, S. Ohzahata, and T. Kato. Towards Practical and Intelligent Routing in Vehicular Ad Hoc Networks. IEEE Transactions on Vehicular Technology, Vol.64, No.12, pp.1-17, 2015.

[15] C. Wu, S. Ohzahata, Y. Ji, T. Kato. How to Utilize Inter-flow Network Coding in VANETs: A Backbone Based Approach. IEEE Transactions on Intelligent Transportation Systems, Vol., No.8, pp.2223-2237, 2016.

[16] B. Moussaoui, S. Djahel, H. Khelifi, and S. Merniz. Towards Enhanced Reactive Routing in Urban Vehicular Ad hoc Networks. ICPE \& NTDS 2015, Paris, France, July 22-24, 2015.

[17] F. K. Karnadi and Z. H. Mo and K. c. Lan. Rapid Generation of Realistic Mobility Models for VANET. Proc. of IEEE Wireless Communications and Networking Conference, pp. 2506-2511, Mar. 2007.

[18] R.A. UZCTEGUI, A. Jose-De-Sucre, G. Acosta-Marum. Wave: A tutorial. IEEE Communications magazine, Vol. 47, No. 5, 2009. 